\author{
K. G. Malyutin, A. A. Revenko
}

\title{
EXTREME PROBLEMS IN THE SPACE OF MEROMORPHIC FUNCTIONS OF FINITE ORDER IN THE HALF-PLANE. II
}

\begin{abstract}
K. G. Malyutin, A. A. Revenko Extreme problems in the space of meromorphic functions of finite order in the half plane. II, Mat. Stud. 54 (2020), 154-161.

The extremal problems in the space of meromorphic functions of order $\rho>0$ in upper half-plane are studed. The method for studying is based on the theory of Fourier coefficients of meromorphic functions. The concept of just meromorphic function of order $\rho>0$ in upper half-plane is introduced. Using Lemma on the Pólya peaks and the Parseval equality, sharp estimate from below of the upper limits of relations Nevanlinna characteristics of meromorphic functions in the upper half plane are obtained.
\end{abstract}

1. Introduction. This paper is a direct continuation of [1]. We shall use, without repeating them, the definitions, results and notation of [1]. However, sections, formulas, theorems and other propositions are labeled independently of [1]. We now state the central results of the present paper.

Let $f \in J M, \lambda=\lambda_{f}$ be the corresponding complete measure of $f, \lambda_{f}:=\lambda=\lambda_{+}-\lambda_{-}$be the Jordan decomposition of $\lambda_{f}$. We set up following notations and terminology

$$
\begin{gathered}
m(r, f):=\frac{1}{r} \int_{0}^{\pi} \log ^{+}\left|f\left(r e^{i \varphi}\right)\right| \sin \varphi d \varphi, \quad \widehat{N}(r, f):=\int_{r_{0}}^{r} \frac{\lambda_{-}(t)}{t^{2}} d t, \\
N(r, f):=\int_{r / 2}^{r} \frac{\lambda_{-}(t)}{t^{3}} d t, \quad T(r, f):=m(r, f)+N(r, f)+m\left(\frac{r}{2}, \frac{1}{f}\right) \text { if } \rho \leq 1, \\
N(r, f):=\int_{r_{0}}^{r} \frac{\lambda_{-}(t)}{t^{3}} d t, \quad T(r, f):=m(r, f)+N(r, f)+m\left(r_{0}, \frac{1}{f}\right), \text { if } \rho \geq 1,
\end{gathered}
$$

where $r_{0}$ is an arbitrary fixed positive number. We have

$$
T(r, f)=T\left(r, \frac{1}{f}\right)
$$

2010 Mathematics Subject Classification: 30D15, 30D30, 30D35.

Keywords: extremal problem; meromorphic function of finite order; complete measure; Pólya lemma; Carleman formula; Nevanlinna characteristic; Parseval equality.

doi:10.30970/ms.54.2.154-161

C) K. G. Malyutin, A. A. Revenko, 2020 
(see $[1,(3)])$. Hence

$$
\int_{0}^{\pi}|\log | f\left(r e^{i \varphi}\right)|| \sin \varphi d \varphi \leq 2 r T(r, f) .
$$

Let us introduce the notions of the order and the indicator of a function. These notions describe growth of a function at infinity.

Definition 1. A function $f \in J M$ is said to be a function of finite order if there exists a positive constant $\beta>1$ such that the inequality $T(r, f) \leq r^{\beta-1}$ is valid for all sufficiently large values of $r$ (i.e $r>r_{0}(\beta)$ ).

The greatest lower bound $\rho$ of such numbers $\beta$ is called the order of the function $f \in J M$.

The space of such functions is denoted by $J M(\rho)$. By $J A(\rho) \subset J M(\rho)$ we denote the subspace of just analytic functions in $\mathbb{C}_{+}$. By $m_{2}(r, f)$ we denote $L_{2}$-norm of the function $f$ about the semicircle of $\left\{r e^{i \theta}: 0 \leq \theta \leq \pi\right\}$ :

$$
m_{2}(r, f)=\left\{\left.\frac{1}{\pi} \int_{0}^{\pi}|\log | f\left(r e^{i \theta}\right)\right|^{2} d \theta\right\}^{1 / 2},
$$

The Fourier coefficients of a function $f \in J M$ are defined as

$$
c_{k}(r, f)=\frac{2}{\pi} \int_{0}^{\pi} \log \left|f\left(r e^{i \theta}\right)\right| \sin k \theta d \theta, \quad k \in \mathbb{N} .
$$

Using (1), we obtain

$$
\left|c_{k}(r, f)\right| \leq \frac{2 k}{\pi} \int_{0}^{\pi}|\log | f\left(r e^{i \varphi}\right) \| \sin \varphi d \varphi \leq \frac{4 r k}{\pi} T(r, f)
$$

and

$$
m(r, f)+m(r, 1 / f)=\int_{0}^{\pi}|\log | f\left(r e^{i \varphi}\right)|| \sin \varphi d \varphi \leq 2 r T(r, f) .
$$

Set

$$
d \lambda_{k}(\zeta)=\frac{\sin k \varphi}{\sin \varphi} \tau^{k-1} d \lambda(\zeta)\left(\zeta=\tau e^{i \varphi}\right), \lambda_{m}(r)=\lambda_{m}(\overline{C(0, r)})
$$

where $\frac{\sin m \varphi}{\sin \varphi}$ is defined for $\varphi=0, \pi$ by continuity.

Note the inequality

$$
\begin{aligned}
\left|\lambda_{k}(r)\right|= & \left|\iint_{\overline{C(0, r)}} d \lambda_{k}(\zeta)\right|=\left|\iint_{\overline{C(0, r)}} \frac{\sin k \varphi}{\sin \varphi} \tau^{k-1} d \lambda(\zeta)\right| \leq \\
& \leq k \iint_{\overline{C(0, r)}} \tau^{k-1} d|\lambda|(\zeta) \leq k r^{k-1}|\lambda|(r) .
\end{aligned}
$$

By Carleman's formula in Grishin's notations [1, (1)],

$$
c_{k}(r, f)=\frac{1}{2^{k}} c_{k}(2 r, f)-\frac{2 r^{k}}{\pi} \int_{r}^{2 r} \frac{\lambda_{k}(t)}{t^{2 k+1}} d t, \quad k \in \mathbb{N} .
$$


The following expressions for the Fourier coefficients for $r>r_{0}$ holds

$$
c_{k}(r, f)=\alpha_{k} r^{k}+\frac{2 r^{k}}{\pi} \int_{r_{0}}^{r} \frac{\lambda_{k}(t)}{t^{2 k+1}} d t, \quad k \in \mathbb{N}
$$

where $\alpha_{k}=r_{0}^{-k} c_{k}\left(r_{0}, v\right)$, and

$$
\begin{gathered}
c_{k}(r, f)=\alpha_{k} r^{k}+\frac{r^{k}}{\pi k r_{0}^{2 k}} \iint_{C_{+}\left(0, r_{0}\right)} \frac{\sin k \varphi}{\operatorname{Im} \zeta} \tau^{k} d \lambda(\zeta)+ \\
+\frac{r^{k}}{\pi k} \iint_{r_{0} \leq|\zeta| \leq r} \frac{\sin k \varphi}{\tau^{k} \operatorname{Im} \zeta} d \lambda(\zeta)-\frac{1}{r^{k} \pi k} \iint \frac{\sin k \varphi}{\operatorname{Im} \zeta} \tau^{k} d \lambda(\zeta), \zeta=\tau e^{i \varphi},
\end{gathered}
$$

(see $\left[1\right.$, formulas (10) and (11)]), where the kernel $\frac{\sin k \varphi}{\tau^{k} \operatorname{Im} \zeta}$ is extended by continuity to the points on the real axis, $\alpha_{k}=r_{0}^{-k} c_{k}\left(r_{0}, v\right)$.

We will use the following lemma.

Lemma 1. Let $f \in J M(\rho), \rho>0$. Then

$$
c_{k}(r, f)=-\frac{2 r^{k}}{\pi} \int_{r}^{\infty} \frac{\lambda_{k}(t)}{t^{2 k+1}} d t, \quad k \in \mathbb{N}, k>[\rho] .
$$

Proof. We divide the equality (5) by $r^{k}, k>\rho$, and pass to the limit as $r \rightarrow \infty$. By inequality (2), the inclusion $f \in J M(\rho)$, we obtain

$$
0=\alpha_{k}+\frac{2}{\pi} \int_{r_{0}}^{\infty} \frac{\lambda_{k}(t)}{t^{2 k+1}} d t, \Rightarrow \alpha_{k}=-\frac{2}{\pi} \int_{r_{0}}^{\infty} \frac{\lambda_{k}(t)}{t^{2 k+1}} d t, \quad k \in \mathbb{N}, k>\rho .
$$

Substituting this value of $\alpha_{k}$ in (5), we obtain (7).

Our main result is the following theorem.

Theorem 1. Let $f \in J M(\rho), \rho>0$. Then

$$
\limsup _{r \rightarrow \infty} \frac{\widehat{N}(r, f)+\widehat{N}(r, 1 / f)}{m_{2}(r, f)} \geq \frac{|\sin \pi \rho|}{\rho(\rho+1)} \sqrt{1 /\left(1-\frac{\sin 2 \pi \rho}{2 \pi \rho}\right)}
$$

and this inequality is sharp, i. e. for some meromorphic function $f, f \in J M(\rho), \rho>0$, equality (8) holds.

Remark 1. In contrast to Theorem 1 and Corollary from [1] which are true for $\rho>1$, this Theorem holds for all $\rho>0$.

2. Proof of Theorem 1. Let us prove Theorem 1. Let $f \in J M(\rho), \rho>0$. Let $\rho$ be a non-integer. In the case of integer $\rho$, the theorem is obvious. 
We define a measure $\tilde{\lambda}$ by the equality $\tilde{\lambda}=\left|\lambda_{f}\right|$. Without loss of generality, we can suppose that the measure $\tilde{\lambda}$ does not load some neighborhood of zero. Denote by $q=[\rho]$, $\beta=\left\{\beta_{k}\right\}$,

$$
\beta_{k}=\left\{\begin{array}{l|l}
\left|\alpha_{k}(f)\right|+\left|\frac{1}{\pi k r_{0}^{2 k}} \iint_{C_{+}\left(0, r_{0}\right)} \frac{\sin k \varphi}{\operatorname{Im} \zeta} \tau^{k} d \lambda_{f}(\zeta)\right|, & 1<k<q+1, \\
-S_{+}(+\infty ; k, \tilde{\lambda}), & k \geq q+1,
\end{array}\right.
$$

where

$$
S_{+}(+\infty ; k, \tilde{\lambda}):=\lim _{r \rightarrow \infty} S_{+}(r ; k, \tilde{\lambda}) .
$$

The following estimate holds

$$
\left|c_{k}(r ; \tilde{\lambda}, \beta)\right| \leq \frac{(k+1) r^{k}}{\pi} \int_{r}^{\infty} \frac{\tilde{\lambda}(t)}{t^{k+2}} d t-\frac{k-1}{r^{k} \pi} \int_{0}^{r} t^{k-2} \tilde{\lambda}(t) d t \leq \frac{A(k+1) r^{\rho+\varepsilon}}{\pi(k-\rho-\varepsilon)}, \quad k \geq q+1,
$$

(see $[1,(20)]$ ), for some $A>0, \varepsilon>0, \rho+\varepsilon<q+1$.

A similar inequality holds for $1 \leq k<q+1$. Thus the pair $(\tilde{\lambda}, \beta)$ is $\rho$-admissible. By Lemma 6 from [1], there exists the function $F \in J A(\rho)$ such that $c_{k}(r, F)=c_{k}(r ; \tilde{\lambda}, \beta)$ for all $r>0$ and for all $k \in \mathbb{N}$.

Set

$$
\widehat{N}_{1}(r)=\widehat{N}_{1}(r, F):=\int_{1}^{r} \frac{\tilde{\lambda}(t)}{t^{2}} d t
$$

The order of the function $\widehat{N}(r)$ is less than or equal to $\rho$. In fact, it is equal $\rho$, because otherwise it follows from Theorem $3[10]$ that this order of $T(r, f)$ is an integer, but we excluded this case.

Integrating by parts in (9), we obtain

$$
\begin{gathered}
\left|c_{k}(r, F)\right| \leq \frac{(k+1) r^{k}}{\pi} \int_{0}^{r} \frac{d \widehat{N}_{1}(t)}{t^{k}}-\frac{k-1}{r^{k} \pi} \int_{0}^{r} t^{k} d \widehat{N}_{1}(t)= \\
=\frac{k}{\pi}\left\{(k-1) \int_{0}^{r}\left(\frac{t}{r}\right)^{k} \frac{\widehat{N}_{1}(t)}{t} d t+(k+1) \int_{r}^{\infty}\left(\frac{r}{t}\right)^{k} \frac{\widehat{N}_{1}(t)}{t} d t\right\}-\frac{2 k}{\pi} r \widehat{N}_{1}(r)
\end{gathered}
$$

for $k \geq q+1$.

By (6), we have

$$
\left|c_{k}(r, F)\right| \leq r^{k}\left(\left|\alpha_{k}\right|+\frac{2}{\pi r_{0}^{2 k}} \int_{0}^{r_{0}} t^{k-1} d \tilde{\lambda}(t)\right)+\frac{1}{\pi} \int_{r_{0}}^{r}\left[\left(\frac{r}{t}\right)^{k}-\left(\frac{t}{r}\right)^{k}\right] \frac{d \tilde{\lambda}(t)}{t},
$$

for $1 \leq k \leq q$.

Therefore, by double integration by parts, we obtain the inequality

$$
\left|c_{k}(r, F)\right| \leq r^{k} \gamma_{k}+\frac{2 k}{\pi} r \widehat{N}_{1}(r)+\frac{k(k-1)}{\pi} \int_{r_{0}}^{r}\left[\left(\frac{r}{t}\right)^{k}-\left(\frac{t}{r}\right)^{k}\right] \frac{\widehat{N}_{1}(t)}{t} d t, 1 \leq k \leq q,
$$


where

$$
\gamma_{k}=\left|\alpha_{k}\right|+\frac{2}{\pi r_{0}^{2 k}} \int_{0}^{r_{0}} t^{k-1} d \tilde{\lambda}(t)+\frac{\tilde{\lambda}\left(r_{0}\right)}{\pi r_{0}^{k+1}} .
$$

Besides, $\left|c_{k}(r, f)\right| \leq\left|c_{k}(r, F)\right|, 1 \leq k \leq q$, and $\left|c_{k}(r, f)\right| \leq-c_{k}(r, F), k \geq q+1$. Hence

$$
m_{2}(r, f) \leq m_{2}(r, F)
$$

Let $\varepsilon>0$ be a fixed number. Applying the lemma of Pólya peaks [11] for functions $\widehat{N}_{1}(r)$, $r^{\rho-\varepsilon}, r^{\rho+\varepsilon}$, we find the sequence $\left(r_{n}\right), \lim _{n \rightarrow \infty} r_{n}=\infty$, ([8, p. 62]) such that

$$
\widehat{N}_{1}(t) \leq\left(\frac{t}{r_{n}}\right)^{\rho-\varepsilon} \widehat{N}_{1}\left(r_{n}\right), 0<t \leq r_{n}, \quad \widehat{N}_{1}(t) \leq\left(\frac{t}{r_{n}}\right)^{\rho+\varepsilon} \widehat{N}_{1}\left(r_{n}\right), t>r_{n} .
$$

Using inequalities (10), (11), (13), we obtain

$$
\begin{gathered}
\left|c_{k}\left(r_{n}, F\right)\right| \leq r_{n}^{k} \gamma_{k}+\frac{2 k}{\pi} \widehat{N}_{1}\left(r_{n}\right)\left(\frac{k^{2}+\rho-\varepsilon}{(\rho-\varepsilon)^{2}-k^{2}}+1\right), \quad 1 \leq k \leq[\rho], \\
\left|c_{k}\left(r_{n}, F\right)\right| \leq \frac{2 k}{\pi} \widehat{N}_{1}\left(r_{n}\right)\left(\frac{k^{2}+\rho-\varepsilon k}{(k-\varepsilon)^{2}-\rho^{2}}-1\right), \quad k>[\rho] .
\end{gathered}
$$

Inequality (13) implies, in particular, that $r_{n}^{[\rho]}=o\left(\widehat{N}_{1}\left(r_{n}\right)\right)$ as $n \rightarrow \infty$, since for $t^{\prime}$ such that $r_{0}<t^{\prime}$ and $\widehat{N}_{1}\left(t^{\prime}\right) \geq\left(t^{\prime}\right)^{\rho-\varepsilon}$ for all $r_{n} \geq t^{\prime}$ the inequality

$$
r_{n}^{q+\varepsilon}<r_{n}^{\rho-\varepsilon} \leq r_{n}^{\rho-\varepsilon} \frac{\widehat{N}_{1}\left(t^{\prime}\right)}{\left(t^{\prime}\right)^{\rho-\varepsilon}} \leq \widehat{N}_{1}\left(r_{n}\right)
$$

holds if $2 \varepsilon<\rho-q$.

From this remark, arbitrariness $\varepsilon$ and (14), it follows that

$$
\limsup _{n \rightarrow \infty} \frac{\left|c_{k}\left(r_{n}, F\right)\right|}{\widehat{N}_{1}\left(r_{n}\right)} \leq \frac{2 k}{\pi} \frac{\rho^{2}+\rho}{\left|\rho^{2}-k^{2}\right|}, \quad k=1,2, \ldots
$$

By the Parseval's equality

$$
\left(m_{2}(r, F)\right)^{2}=\frac{1}{2} \sum_{k=-\infty}^{\infty}\left|c_{k}(r, F)\right|^{2},
$$

we obtain the inequality

$$
\liminf _{n \rightarrow \infty} \frac{m_{2}(r, F)}{\widehat{N}_{1}(r)} \leq\left\{\sum_{k=-\infty}^{\infty} \frac{2 k^{2}}{\pi^{2}} \frac{\left(\rho^{2}+\rho\right)^{2}}{\left|\rho^{2}-k^{2}\right|^{2}}\right\}^{1 / 2} .
$$

The sum on the right-hand side of this inequality can be easily found using residues [12]. It is equal

$$
\sum_{k=-\infty}^{\infty} \frac{2 k^{2}}{\pi^{2}} \frac{\left(\rho^{2}+\rho\right)^{2}}{\left|\rho^{2}-k^{2}\right|^{2}}=\left(\frac{\rho(\rho+1)}{|\sin \pi \rho|}\right)^{2}\left(1-\frac{\sin 2 \pi \rho}{2 \pi \rho}\right) .
$$


Since $\widehat{N}_{1}(r)=\widehat{N}(r, f)+\widehat{N}(r, 1 / f)$, by (15) we get the statement of Theorem 1 .

We show that the estimate (8) is sharp.

Example. The function

$$
f(z)=\exp \left\{\frac{1}{\pi i} \int_{t \geq 1} \frac{(t z+1)^{q+1} t^{\rho}}{(t-z)\left(t^{2}+1\right)^{q+1}} d t\right\}, \quad \rho>0, q=[\rho]
$$

is the analytic function in $\mathbb{C}_{+}$with the complete measure $\lambda_{f}(t)=t^{\rho+1}=\lambda_{+}(t)=\tilde{\lambda}(t)$ if $t \in \mathbb{R}, t \geq 1$, and $d \lambda_{f}(z) \equiv 0$ if $z \in \mathbb{C}_{+} \cup(-\infty, 1)$,

$$
N_{1}(r)=N(r, 1 / f)=\int_{1}^{r} t^{\rho-2} d t=\frac{r^{\rho-1}}{\rho-1}-\frac{1}{\rho-1}, N(r, f) \equiv 0, \lambda_{k}(t)=\frac{k(\rho+1)}{k+\rho} t^{k+\rho} .
$$

Now estimate the function

$$
u(z)=\operatorname{Re}\left\{\frac{1}{\pi i} \int_{t \geq 1} \frac{(t z+1)^{q+1} t^{\rho}}{(t-z)\left(t^{2}+1\right)^{q+1}} d t\right\} .
$$

We have

$$
u(z)=\frac{1}{\pi i} \int_{t \geq 1}=\frac{1}{\pi i} \int_{1 \leq t \leq \frac{1}{2}|z|}+\frac{1}{\pi i} \int_{\frac{1}{2}|z|<t \leq 2|z|}+\frac{1}{\pi i} \int_{t>2|z|}=I_{1}+I_{2}+I_{3} .
$$

Further

$$
\begin{aligned}
\left|I_{1}\right| & \leq \frac{1}{\pi} \int_{1 \leq t \leq \frac{1}{2}|z|} \frac{(t|z|+1)^{q+1} t^{\rho}}{\left(\frac{1}{2}|z|\right)\left(t^{2}+1\right)^{q+1}} d t \leq \frac{1}{\pi} \int_{t \geq 1} \frac{d t}{t^{1+\varepsilon}} \times \max _{1 \leq t \leq \frac{1}{2}|z|} \frac{t^{\rho+1+\varepsilon}(t|z|+1)^{q+1}}{\left(\frac{1}{2}|z|\right)\left(t^{2}+1\right)^{q+1}} \leq C_{q, \varepsilon}^{(1)}|z|^{\rho+\varepsilon}, \\
\left|I_{3}\right| & \leq \frac{1}{\pi} \int_{2|z|}^{\infty} \frac{(t|z|+1)^{q+1} t^{\rho}}{\left(\frac{1}{2} t\right)\left(t^{2}+1\right)^{q+1}} d t \leq \frac{1}{\pi} \int_{t \geq 1} \frac{d t}{t^{1+\varepsilon}} \times \max _{t \geq 2|z|} \frac{t^{\rho+1+\varepsilon}(t|z|+1)^{q+1}}{\left(\frac{1}{2} t\right)\left(t^{2}+1\right)^{q+1}} \leq C_{q, \varepsilon}^{(2)}|z|^{\rho+\varepsilon} \\
I_{2} & =\frac{1}{\pi i} \int_{\frac{1}{2}|z|<t \leq 2|z|} \frac{(t z+1)^{q+1}-\left(t^{2}+1\right)^{q+1}}{\left(t^{2}+1\right)^{q+1}(t-z)} t^{\rho} d t+\frac{1}{\pi i} \int_{\frac{1}{2}|z|<t \leq 2|z|} \frac{t^{\rho} d t}{t-z}=I_{2}^{(1)}+I_{2}^{(2)} .
\end{aligned}
$$

As can be easily seen,

$$
\left|\frac{(t z+1)^{q+1}-\left(t^{2}+1\right)^{q+1}}{(t-z)}\right| \leq C_{q}|z|^{2 q+1}
$$

for $\frac{1}{2}|z|<t \leq 2|z|$ (since the expression in the left-hand side is a polynomial in both $z$ and $t$ ). Therefore

$$
\left|I_{2}^{(1)}\right| \leq \frac{C_{q}}{\pi} \int_{\frac{1}{2}|z|<t \leq 2|z|} \frac{|z|^{2 q+1} t^{\rho}}{\left(t^{2}+1\right)^{q+1}} d t \leq C_{q, \varepsilon}^{(3)}|z|^{\rho+\varepsilon} \int_{1}^{\infty} \frac{d t}{t^{1+\varepsilon}}
$$

Finally, by the Poisson formula for the half-plane, we obtain

$$
\operatorname{Re} I_{2}^{(2)}=\frac{1}{\pi} \int_{\frac{1}{2}|z|<t \leq 2|z|} t^{\rho} \operatorname{Im} \frac{1}{t-z} d t \leq 2^{\rho}|z|^{\rho} .
$$


Thus,

$$
\log |f(z)|<C_{q, \varepsilon}|z|^{\rho+\varepsilon} .
$$

Therefore

$$
m(r, f)<C_{q, \varepsilon} \pi r^{\rho+\varepsilon-1} .
$$

Since $N(r, f) \equiv 0$ we have

$$
T(r, f)=m(r, f)<C_{q, \varepsilon} \pi r^{\rho+\varepsilon-1} .
$$

Hence $f \in[\rho, \infty]^{+}$.

By (7), we obtain

$$
c_{k}(r, f)=-\frac{2 r^{k} k(\rho+1)}{\pi(k+\rho)} \int_{r}^{\infty} t^{\rho-k-1} d t=\frac{2 r^{\rho} k(\rho+1)}{\pi\left(k^{2}-\rho^{2}\right)}, \quad k>[\rho] .
$$

Then

$$
\frac{c_{k}(r, f)}{\widehat{N}(r, 1 / f)}=\frac{2 r^{\rho} k\left(\rho^{2}-1\right)}{\pi\left(k^{2}-\rho^{2}\right)}+o(1), \quad r \rightarrow \infty, k>[\rho] .
$$

By (6), we obtain further

$$
\begin{aligned}
& c_{k}(r, f)=r^{k} \tilde{\gamma}+\frac{\rho+1}{\pi} \int_{r_{0}}^{r}\left[\left(\frac{r}{t}\right)^{k}-\left(\frac{t}{r}\right)^{k}\right] t^{\rho-1} d t= \\
& =r^{k} \tilde{\gamma}+\frac{2 r^{\rho} k\left(\rho^{2}-1\right)}{\pi\left(k^{2}-\rho^{2}\right)}+o(1), \quad r \rightarrow \infty, 1 \leq k \leq[\rho],
\end{aligned}
$$

where

$$
\tilde{\gamma}=\alpha_{k}+\frac{2}{\pi r_{0}^{2 k}} \int_{0}^{r_{0}} t^{k-1} d \tilde{\lambda}(t)
$$

From this and (16), we obtain

$$
\lim _{r \rightarrow \infty} \frac{c_{k}(r, f)}{\widehat{N}(r, 1 / f)}=\frac{2 k\left(\rho^{2}-1\right)}{\pi\left(k^{2}-\rho^{2}\right)}, \quad k \in \mathbb{N} .
$$

Thus, for $f(z)$ the estimate (8) is exact.

Acknowledgment. The reported study was funded by RFBR according to the research project No 18-01-00236.

\section{REFERENCES}

1. K.G. Malyutin, A.A. Revenko, Extreme problems in the space of meromorphic functions of finite order in the half-plane, Mat. Stud., 52 (2019), 144-155. doi:10.30970/ms.52.2.144-155

2. L.A. Rubel, B.A. Taylor, A Fourier series method for meromorphic and entire function, Bull. Soc. Math. France, 96 (1968), 53-96. 
3. A.A. Kondratyuk, The Fourier series method for entire and meromorphic functions of completely regular growth. I, Mat. Sb., 106 (1978), №148, 386-408; English transl. in Math. USSR-Sb., 35 (1979).

4. A.A. Kondratyuk, The Fourier series method for entire and meromorphic functions of completely regular growth. II, Mat. Sb., 113 (1980), №155, 118-132; English transl. in Math. USSR-Sb. 41 (1982).

5. A.A. Kondratyuk, The Fourier series method for entire and meromorphic functions of completely regular growth. III, Mat. Sb., 120 (1983), №162, 331-343; English transl. in Math. USSR-Sb., 48 (1984).

6. K.G. Malyutin, Fourier series and $\delta$-subharmonic functions of finite $\gamma$-type in a half-plane, Sbornik: Mathematics, 192 (2001), №6, 843-861. doi:10.1070/SM2001v192n06ABEH000572

7. J.B. Miles, D.P. Shea, An extremal problem in value distribution theory, Quart. J. Math. Oxford, 24 (1973), 377-383.

8. A.A. Kondratyuk, Fourier Series and Meromorphic Functions, Vyshcha shkola, Lviv, 1988. (in Russian)

9. M.A. Fedorov, A.F. Grishin, Some questions of the Nevanlinna theory for the complex half-plane, Mathematical Physics, Analysis and Geometry, 1 (1998), №3, 223-271.

10. K.G. Malyutin, N. Sadik, Representation of subharmonic functions in a half-plane, Sbornik: Mathematics, 198 (2007), №12, 1747-1761. doi:10.1070/SM2007v198n12ABEH00390

11. G. Pólya, Untersuchungen über Lücen and Singularitätin von Potenzreihen, Math. Zeitschrift, 29 (1929), 549-640.

12. J. Bak, D.J. Newman, Complex Analysis, Springer, 2010.

Kursk State University, Department of Mathematical Analysis

Kursk, Russia

malyutinkg@gmail.com

revenko253@mail.ru 